\title{
miR-29 mediates exercise-induced skeletal muscle angiogenesis by targeting VEGFA, COL4A1 and COL4A2 via the PI3K/Akt signaling pathway
}

\author{
LEI CHEN $^{1}$, JUN BAI ${ }^{2}$ and YANFEI $\mathrm{LI}^{3}$ \\ ${ }^{1}$ Department of Physical Education, Shanghai Jiaotong University, Shanghai 200240; ${ }^{2}$ Institute of Immunology, Shanghai \\ Jiao Tong University School of Medicine, Shanghai 200025; ${ }^{3}$ Office of Academic Research, Shanghai University of \\ Medicine and Health Sciences Affiliated Zhoupu Hospital, Shanghai 201318, P.R. China
}

Received June 26, 2019; Accepted March 12, 2020

DOI: $10.3892 / \mathrm{mmr} .2020 .11164$

\begin{abstract}
The present study investigated the molecular changes and related regulatory mechanisms in the response of skeletal muscle to exercise. The microarray dataset 'GSE109657' of the skeletal muscle response to high-intensity intermittent exercise training (HIIT) was downloaded from the Gene Expression Omnibus database. Differentially expressed genes (DEGs) were screened and analyzed using weighted gene co-expression network analysis (WGCNA) to identify the significant functional co-expressed gene modules. Moreover, functional enrichment analysis was performed for the DEGs in the significant modules. In addition, protein-protein interaction (PPI) network and microRNA (miR)-transcription factor (TF)-target regulatory network were constructed. A total of 530 DEGs in the skeletal muscle were screened after HIIT, suggesting an effect of HIIT on the skeletal muscle. Moreover, three significant modules (brown, blue and red modules) were identified after WGCNA, and the genes Collagen Type IV $\alpha 1$ Chain (COL4A1) and COL4A2 in the brown module showed the strongest correlation with HIIT. The DEGs in the three modules were significantly enriched in focal adhesion, extracellular matrix organization and the PI3K/Akt signaling pathway. Furthermore, the PPI network contained 104 nodes and 211 interactions. Vascular endothelial growth factor A (VEGFA), COL4A1 and COL4A2 were the hub genes in the PPI network, and were all regulated by $\mathrm{miR}-29 \mathrm{a} / \mathrm{b} / \mathrm{c}$. In addition, VEGFA, COL4A1 and COL4A2 were significantly upregulated in the skeletal muscle response to HIIT. Therefore, the present results suggested that the growth and migration of
\end{abstract}

Correspondence to: Dr Yanfei Li, Office of Academic Research, Shanghai University of Medicine and Health Sciences Affiliated Zhoupu Hospital, 1500 Zhouyuan Road, Shanghai 201318, P.R. China

E-mail: fenny_YL@163.com

Key words: skeletal muscle, high-intensity intermittent exercise training, angiogenesis vascular endothelial cells, and skeletal muscle angiogenesis may be regulated by miR-29a/b/c targeting VEGFA, COL4A1 and COL 4A 2 via the PI3K/Akt signaling pathway. The present results may provide a theoretical basis to investigate the effect of exercise on skeletal muscle.

\section{Introduction}

Skeletal muscle accounts for $\sim 42 \%$ of the total body mass in males and $36 \%$ in females (1); it is a metabolically active tissue and is responsible for $30 \%$ of the resting metabolic rate in adults (2). Apart from skeletal motion, skeletal muscle plays key roles in calorigenesis, blood glucose control, metabolic balance and the support and protection of soft tissue (1). Exercise training has the ability to improve pathological conditions involving metabolic disorders and prevent various lifestyle-related chronic maladies, partly due to its regulation of metabolic homeostasis and the molecular responses of skeletal muscle (2). Moreover, exercise induces various adaptive responses in the skeletal muscle, including mitochondrial biogenesis (3), lipid metabolism (4), glycometabolism (5) and ultrastructural changes (6).

Baar et al (7), reported that mitochondrial biogenesis triggered by exercise is associated with the increase of the transcriptional coactivators peroxisome proliferator-activated receptor $\gamma$ coactivator-1 (PGC-1), nuclear respiratory factor 1 (NRF-1) and NRF-2. Cantó et al (8), revealed that AMP-activated protein kinase (AMPK) is first activated during the adaptive responses in skeletal muscle after exercise, while sirtuin 1 (SIRT1) is activated with deficient AMPK activity, suggesting an acetylation regulation mechanism of the AMPK/SIRT1 axis. High-intensity intermittent exercise training (HIIT) improves the skeletal myopathy in patients with heart failure associated with the increased expression of the insulin-like growth factor 1 bioregulation system (9). Exercise training can induce the increased expression level of cytokines secreted by skeletal muscle cells, including IL-6, IL-1 and IL-10, which have anti-inflammatory effects (10). In addition, microRNAs (miRNAs/miRs), a class of non-coding small RNAs regulating genes at a post-transcriptional level, also play crucial roles in the skeletal muscle response to exercise $(1,11)$. 
The expression level of miR-761 is reduced in the mouse skeletal muscle response to exercise and its overexpression inhibits the P38 mitogen-activated protein kinase signaling pathway and PGC-1 $\alpha$, which are associated with mitochondrial biogenesis (12). Although previous studies have been conducted, the specific molecular mechanisms of mouse skeletal muscle response to exercise are not fully understood $(13,14)$. Therefore, the present study investigated the molecular changes and related regulatory mechanisms in skeletal muscle response to exercise.

The microarray dataset 'GSE109657' of the skeletal muscle response to HIIT used in the present study was contributed by Miyamoto-Mikami et al (15). These authors investigated the differentially expressed genes (DEGs) and the associated functions, and significantly upregulated DEGs are found to be associated with glucose metabolism and mitochondrial membranes (15). In the present study, DEGs were identified and analyzed using weighted gene co-expression network analysis (WGCNA), which is effective for the identification of functional co-expressed gene modules (16). In addition, except for the functional enrichment analysis, miRNAs and transcription factors (TFs) were predicted in order to construct the miRNA-TF-target regulatory network. Thus, the present results may provide a theoretical basis for the investigation of the effect of exercise on skeletal muscle.

\section{Materials and methods}

Microarray data. The 'GSE109657' gene expression dataset of human skeletal muscle was downloaded from the Gene Expression Omnibus (GEO) database (https://www.ncbi.nlm. nih.gov/geo/). There were 22 biopsy samples in this dataset, which were collected from the vastus lateralis muscle of 11 young and healthy men before (GSM2948027-GSM2948037) and after (GSM2948038-GSM2948048) a 6-week HIIT. The platform of this dataset was GPL16686 [HuGene-2_0-st] Affymetrix Human Gene 2.0 ST Array [transcript (gene) version]. Since the dataset was obtained from a public database, no ethical approval was obtained in the present study.

Data preprocessing and screening of DEGs. The Oligo in $\mathrm{R}$ package (v.1.34.0; http://bioconductor.org/help/search/index. html?q=oligo/) was used to perform raw data preprocessing, including format conversion, missing value supplement, background correction and data standardization. The probes were annotated according to the annotation file on the platform and were removed when the gene symbol did not match. The differentially expressed analysis among samples was performed utilizing the classical Bayes method in limma package (R v.3.3.3) (17) and the DEGs were screened with the threshold of $\mathrm{P}<0.05$ and llog fold change (FC) $\mid>0.263$.

WGCNA for DEGs. WGCNA (http://www.inside-r. org/packages/cran/WGCNA/docs/bicor) was used to identify the modules and genes associated with HIIT based on the expression level of DEGs, and the DEGs were clustered into different modules according their co-expression relationships. WGCNA was conducted according to a previous study by Langfelder and Horvath (18), including the definition of gene co-expression matrix $S_{m m}=\left|\operatorname{cor}_{(m, n)}\right|$, the definition of adjacent function $\mathrm{a}_{\mathrm{mn}}=\operatorname{power}_{(\mathrm{Smn})}$, the determination of weighted coef- ficient $\beta(\geq 0.8)$ and the measurement of dissimilarity between nodes. The minimum number of genes in each module was set as 20 and the cluster analysis height of the module was set as 0.2 in the identification of gene modules. In addition, the module significance was calculated to identify the correlation between modules and HIIT.

Functional enrichment analysis for the genes in significant modules. The online database for annotation, visualization and integrated discovery tool (v.6.8; https://david-d.ncifcrf.gov/) was used to investigate the function of the genes in significant modules, including biological processes in Gene Ontology (GO $\mathrm{BP}$ ) and the Kyoto Encyclopedia of Genes and Genomes (KEGG) signaling pathways. The number of enrichment genes was set as count $\geq 2$ and $\mathrm{P}<0.05$ was selected as the threshold.

Construction of protein-protein interaction network. The genes in significant modules were integrated and uploaded to the STRING database (version: 10.0; http://www.string-db. org/) to retrieve the protein-protein interactions (PPIs) with the following parameters: Species was set as human and the PPI score was set as 0.4 (median confidence). Cytoscape software (v.3.2.0; http://www.cytoscape.org/) was used to construct the visualized PPI network based on the retrieved interactions from STRING. A high node degree centrality value indicated the hub nodes in the PPI network (19).

Construction of miRNA-TF-target regulatory network. The over-representation analysis method of enrichment in WebGestalt (v.2017; http://www.webgestalt.org/) was used to predict the miRNA-target interactions and TF-target interactions for the genes with node degree $>5$ in the PPI network. $\mathrm{P}<0.05$ was selected as the threshold. In addition, Cytoscape software was used to construct the miRNA-TF-target regulatory network with the significantly enriched miRNA-target interactions and TF-target interactions.

Confirmatory analysis. In order to investigate the expression and function of the DEGs, the 'GSE41769' gene expression dataset of human skeletal muscle, which was contributed by Catoire et al (20), was downloaded from the GEO database. This dataset included 36 skeletal muscle biopsy samples, which were collected from both the legs of nine healthy middle-aged men before and after $1 \mathrm{~h}$ of one-legged exercise. The platform of this dataset was GPL11532 [HuGene-1_1-st] Affymetrix Human Gene 1.1 ST Array [transcript (gene) version]. Data were preprocessed and differentially expressed analysis was performed using the method mentioned above, and the DEGs were screened within the threshold of $\mathrm{P}<0.05$. Moreover, functional enrichment analysis was also conducted for these DEGs using the method described above.

\section{Results}

Data preprocessing and screening of DEGs. The gene expression in each sample was at the same level after data normalization, suggesting that they could be used in the subsequent analyses (Fig. 1A). A total of 530 DEGs were screened in the vastus lateralis muscle after HIIT, of which 209 genes were significantly upregulated, while 321 genes were significantly 

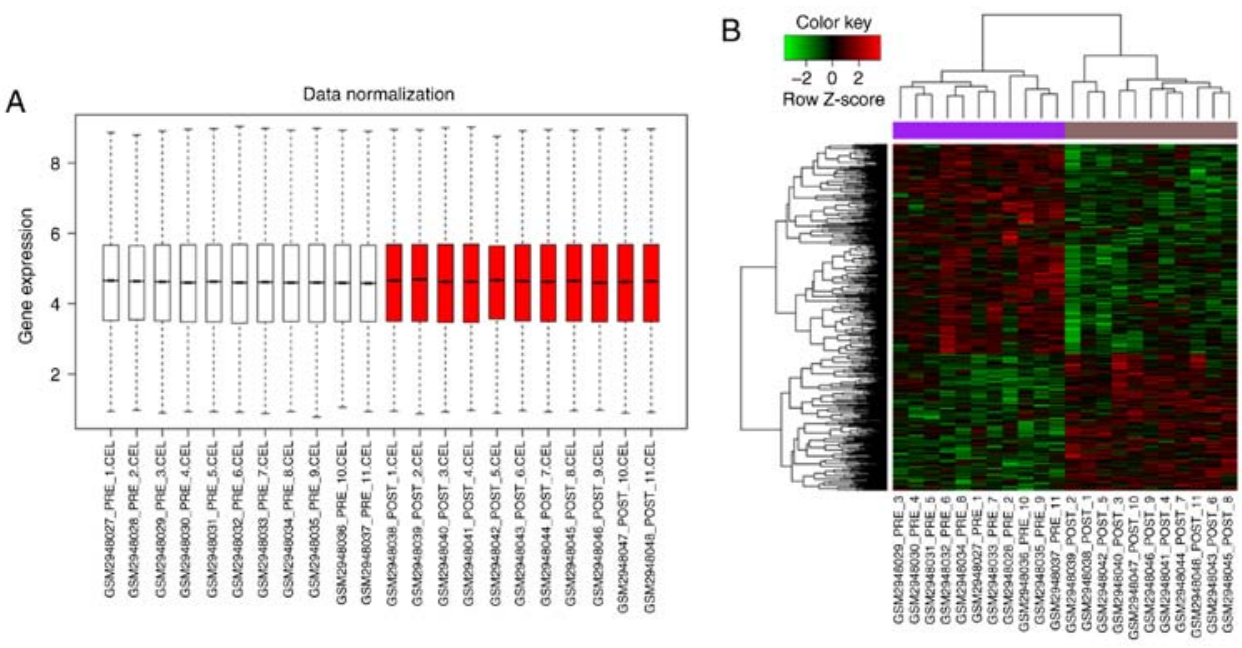

Figure 1. Results of differential expression analysis. (A) Boxplot of the levels of gene expression in each sample after data normalization. (B) Heatmap of differentially expressed genes before or after 6-week high-intensity intermittent exercise training.
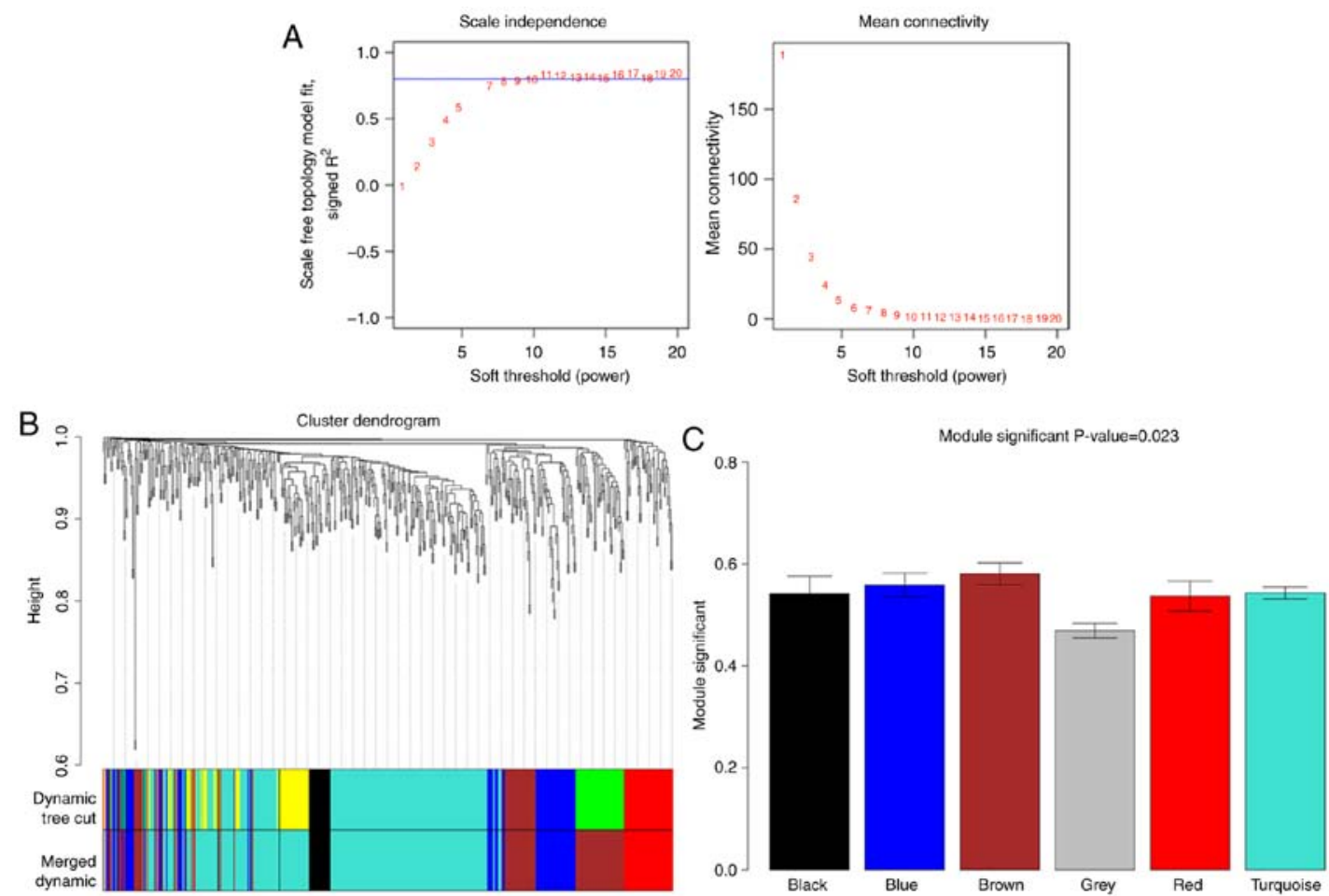

Figure 2. Weighted gene co-expression network analysis. (A) Selection diagram of adjacency matrix weight parameter 'power'. (B) Identification of gene system clustering tree under dissimilarity matrix using dynamic hybrid shearing algorithm. (C) High-intensity intermittent exercise training correlated co-expression modules.

downregulated. The heatmap of DEGs displayed in Fig. 1B indicated that DEGs could be distinguished in the muscle biopsy samples before and after HIIT.

WGCNA for DEGs. The value of the power parameter in the adjacent function was determined as eight. A total of six co-expression modules were identified for the DEGs with absolute correlation $\geq 0.5$, of which three modules had the absolute correlation $\geq 0.8$; these were the brown, blue and red modules. The DEGs in these three modules were used in the following analysis (Table I). The DEGs in the brown module showed the strongest correlation with HIIT and those in the
Table I. High-intensity intermittent exercise training correlated co-expression modules.

\begin{tabular}{lcr}
\hline Module & Correlation coefficient & \multicolumn{1}{c}{ P-value } \\
\hline MEbrown & 0.86 & $2.35 \times 10^{-7}$ \\
MEblue & -0.81 & $5.94 \times 10^{-6}$ \\
MEred & -0.8 & $9.49 \times 10^{-6}$ \\
MEturquoise & -0.79 & $1.39 \times 10^{-5}$ \\
MEblack & -0.68 & 0.0004783 \\
MEgrey & -0.63 & 0.001525 \\
\hline
\end{tabular}



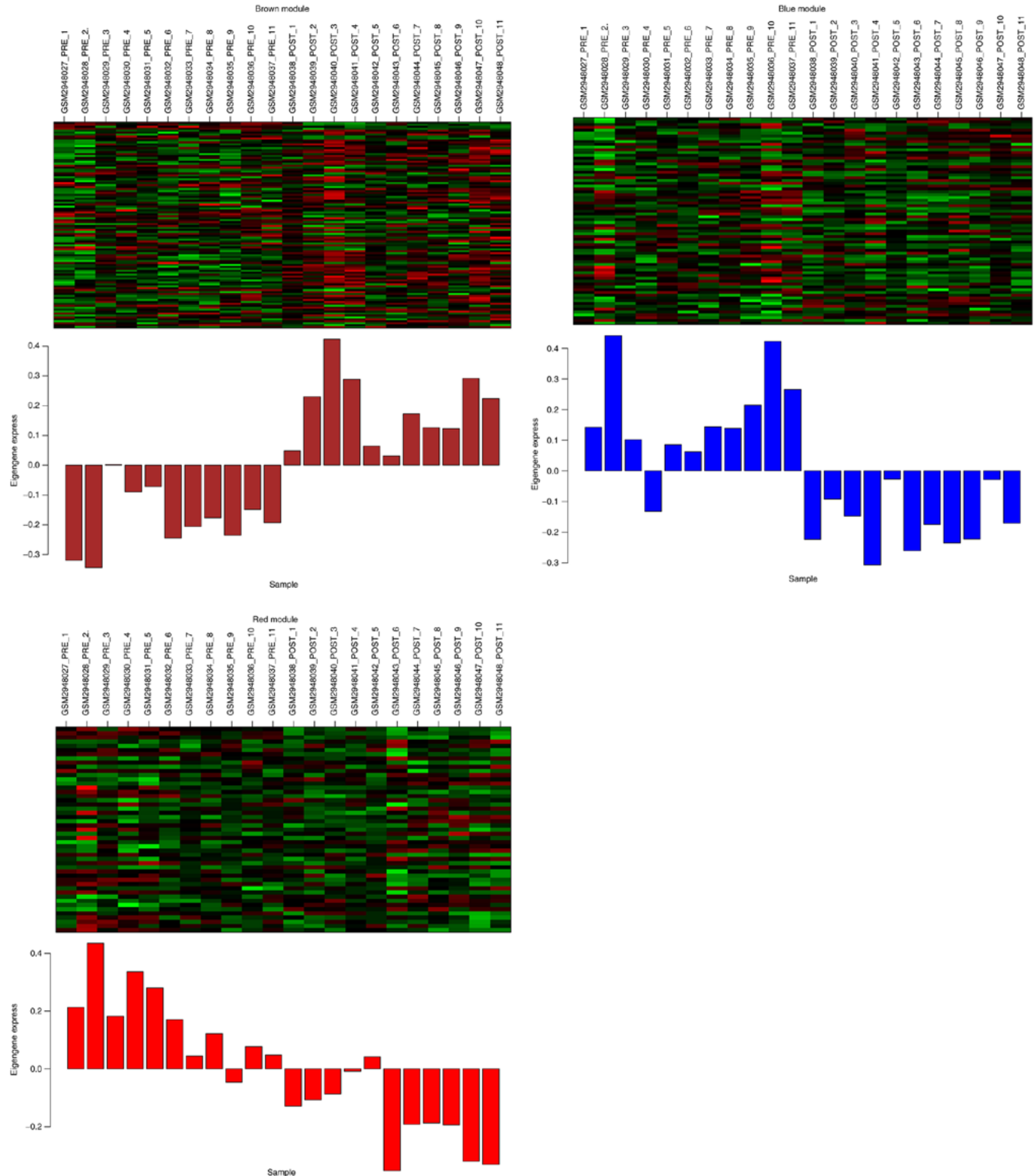

Figure 3. In total, three significant modules (brown, blue and red module) are identified after weighted gene co-expression network analysis. Heatmaps of the differentially expressed genes in the brown, blue and red modules were displayed.

grey module were not clustered into co-expression modules with other DEGs (Fig. 2).

Expression levels of the genes in significant modules. In total, three significant modules (brown, blue and red modules) were identified after WGCNA, and a total of 106, 74 and 49 DEGs were included in the brown, blue and red module, respectively. Fig. 3 shows the heatmap of the DEGs in each of the three modules.
Functional enrichment analysis for the genes in significant modules. The results of functional enrichment analysis suggested that the DEGs in the brown module were significantly enriched in eight KEGG signaling pathways and 14 GO_BPs, such as 'hsa04510:Focal adhesion' [involving collagen type IV $\alpha 1$ (COL4A1) and COL4A2], 'hsa04151:PI3K-Akt signaling pathway' (involving COL4A1 and COL4A2), 'GO:0030198 extracellular matrix organization' (involving COL4A1 and COL4A2) and 'GO:0038063 collagen-activated tyrosine 
A

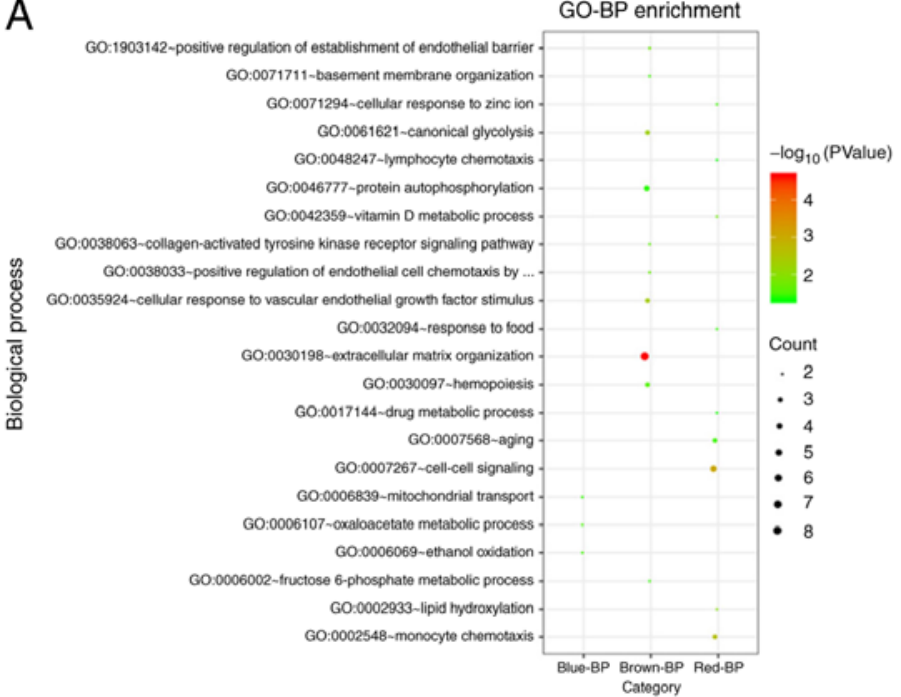

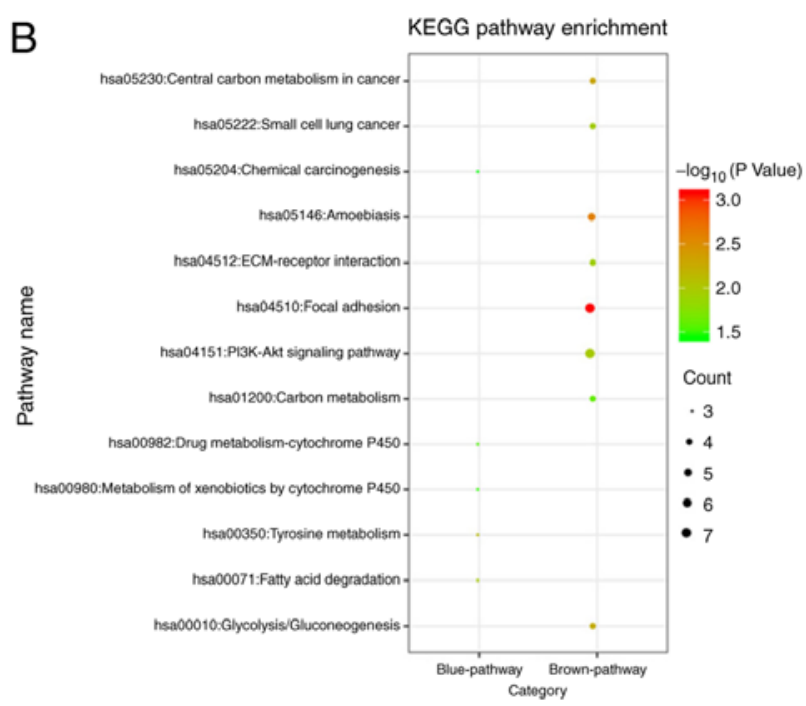

Figure 4. Significantly enriched KEGG signaling pathway and top ten GO_BPs for the differentially expressed genes in the brown, blue and red modules. (A) Top ten GO_BP terms for the differentially expressed genes in the brown, blue and red modules. (B) KEGG signaling pathway for the differentially expressed genes in the brown, blue and red modules. KEGG, Kyoto Encyclopedia of Genes and Genomes; GO_BP, biological processes in Gene ontology.

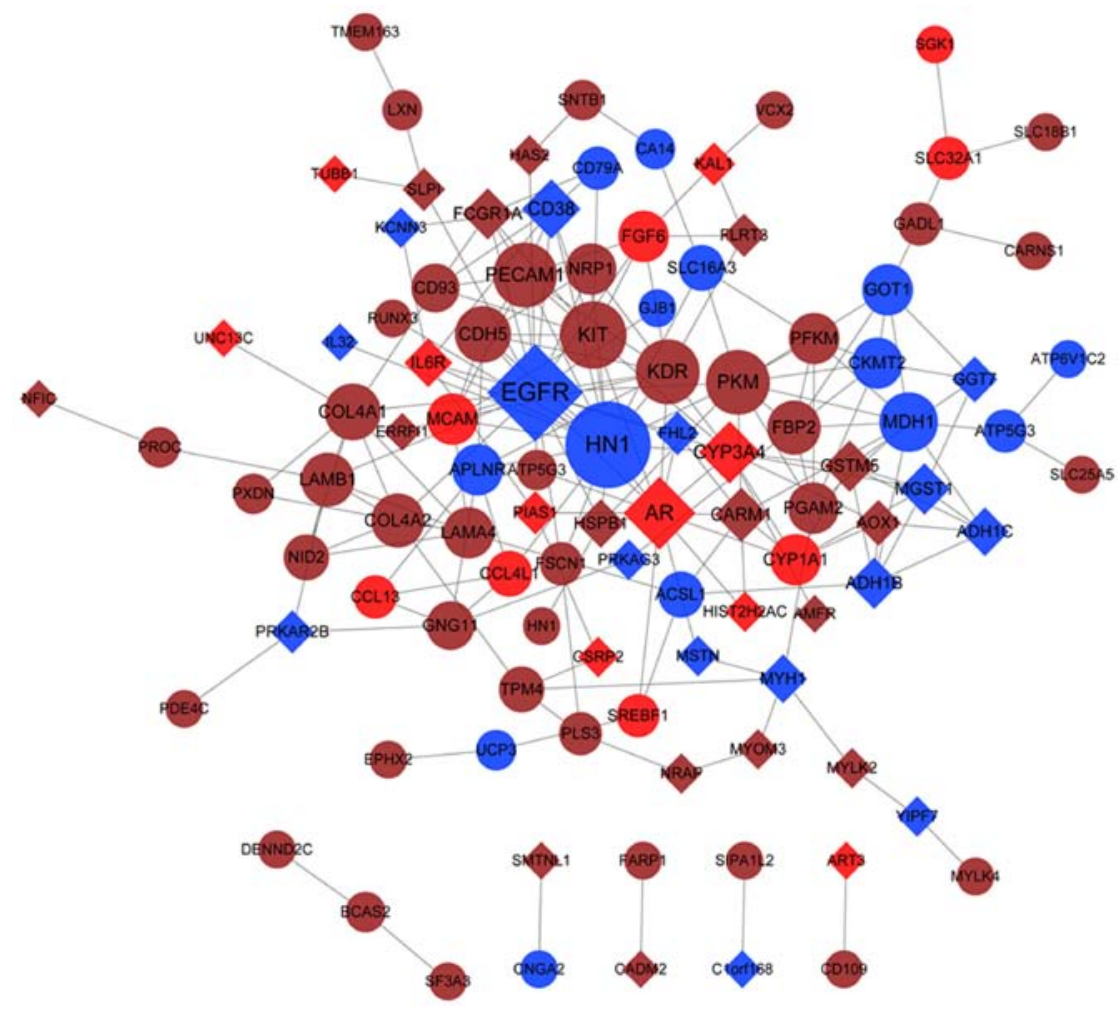

Figure 5. Protein-protein interaction network for the DEGs in the brown, blue and red modules. A circle represents upregulated DEGs; a rhombus represents downregulated DEGs. Brown, blue and red represent the DEGs belonging to the brown, blue and red modules, respectively. DEGs, differentially expressed genes.

kinase receptor signaling pathway' (involving COL4A1 and COL4A2). The DEGs in the blue module were significantly enriched in five KEGG signaling pathways and three GO_BPs, such as 'hsa00350:Tyrosine metabolism' [involving alcohol dehydrogenase $1 \mathrm{C}, \gamma$ polypeptide (ADH1C), alcohol dehydrogenase 1B and $\beta$ polypeptide (ADH1B)], 'hsa00071:Fatty acid degradation' (involving ADH1C and ADH1B) and 'GO:0006107 oxaloacetate metabolic process' (involving glutamic-oxaloacetic transaminase 1 and malate dehydro- genase 1). The DEGs in the red module were significantly enriched in nine GO_BPs, including 'GO:0007267 cell-cell signaling' [involving fibroblast growth factor 6 (FGF6) and androgen receptor (AR)], 'GO:0002548 monocyte chemotaxis' (involving C-C motif chemokine ligand 13 and C-C motif chemokine ligand 4 like 1) and others (Fig. 4). It was found that no KEGG pathways were significantly enriched for the DEGs in the red module. The significantly enriched KEGG pathways and top ten GO_BPs are presented in Fig. 4, 


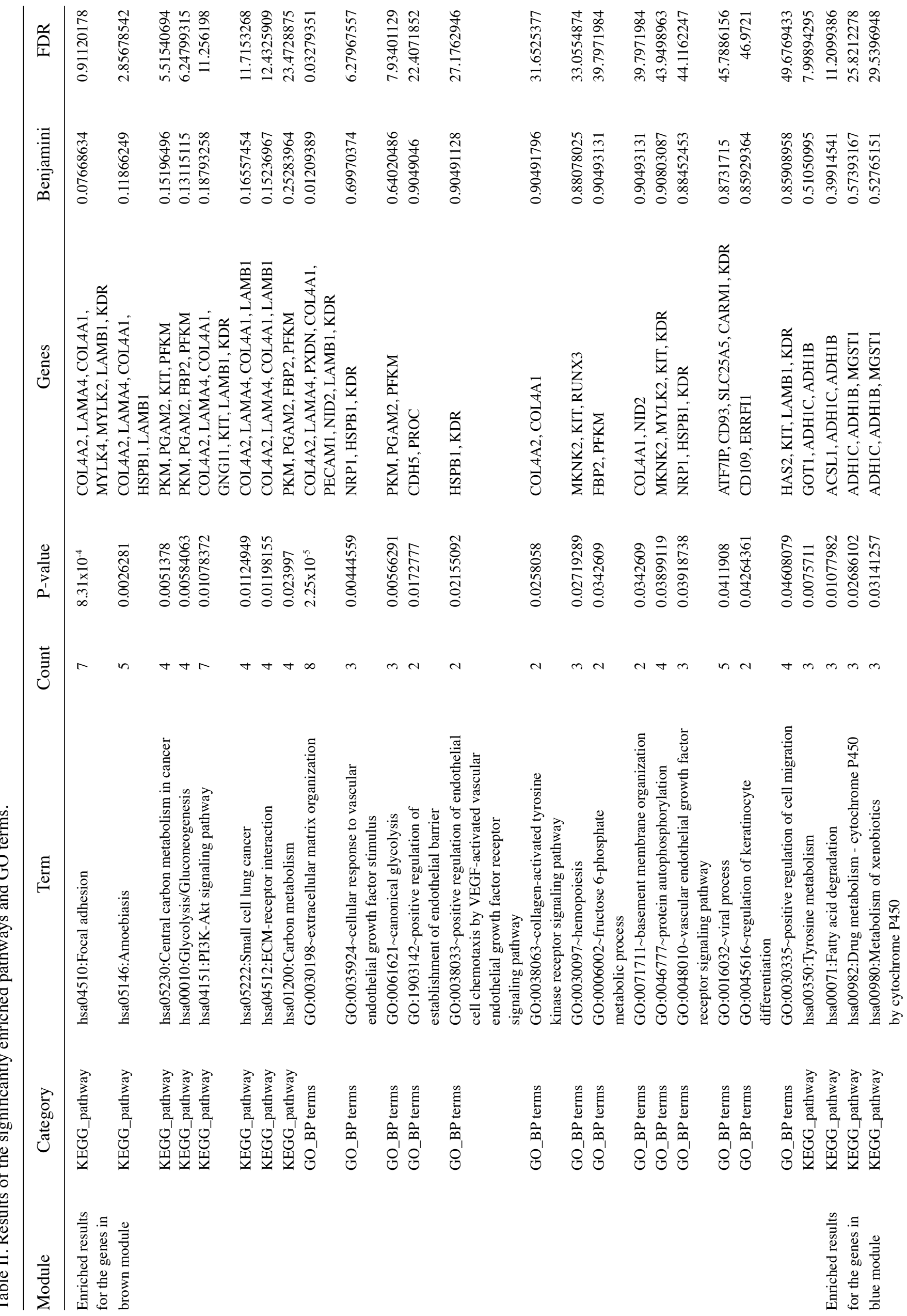




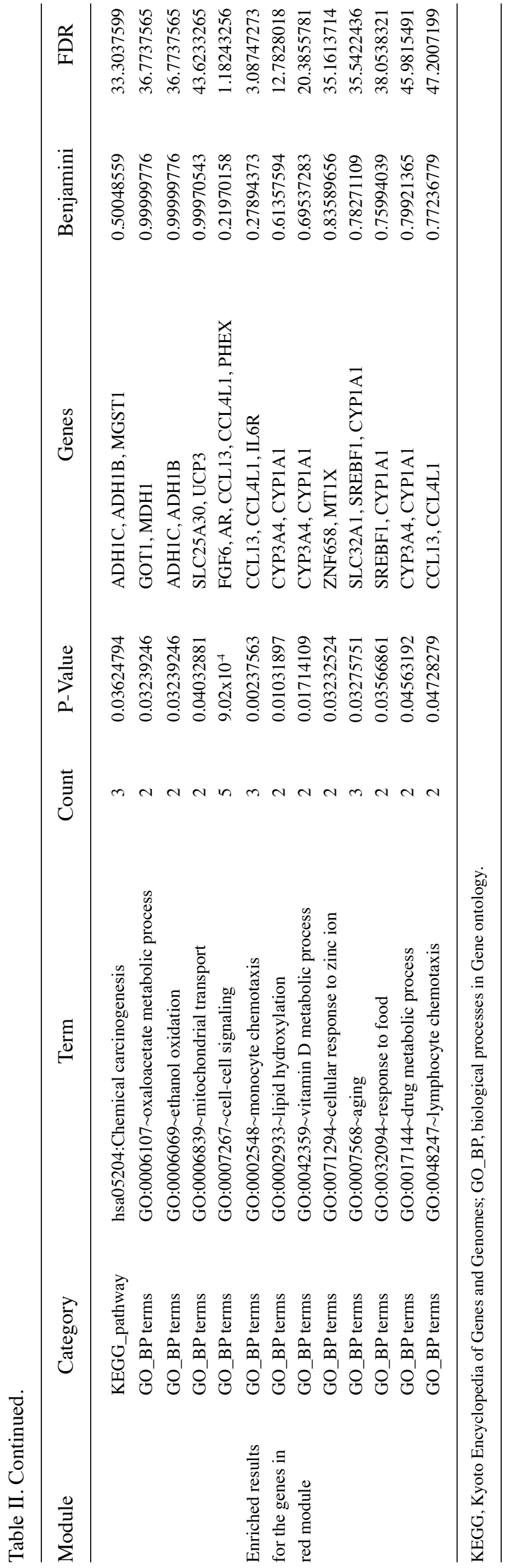

and detailed information of significantly enriched results is shown in Table II.

Construction of PPI network. The PPI network contained 104 nodes, of which 57 belonged to the brown module, 29 belonged to the blue module and 18 belonged to the red module, and 211 interactions (Fig. 5). The nodes in the PPI network with a degree of $>5$ are presented in Table III. It was demonstrated that epidermal growth factor receptor (EGFR, degree =23), vascular endothelial growth factor A (VEGFA), $\mathrm{AR}$, proto-oncogene receptor tyrosine kinase (KIT), COL4A1 (degree =8) and COL4A2 (degree =7) were the hub genes with higher degrees in the PPI network. Furthermore, EGFR and VEGFA were the genes in the blue module, and AR was the gene in the red module, while KIT, COL4A1 and COL4A2 were the genes in the brown module (Fig. 5).

Construction of miRNA-TF-target regulatory network. The miRNA-TF-target regulatory network included 27 nodes and 36 regulatory interactions (Fig. 6). In total, five miRNAs and eight TFs were predicted to regulate 14 DEGs, including 12 upregulated DEGs and two downregulated DEGs. It was found that VEGFA, COL4A1, COL4A2 and FGF6 were the hub nodes in the regulatory network, in which VEGFA, COL4A1 and COL4A2 were all regulated by miR-29a/b/c; $\mathrm{miR}-29 \mathrm{a} / \mathrm{b} / \mathrm{c}$ regulated only these three DEGs in this network. Moreover, FGF6 was regulated by five TFs, including interferon consensus sequence binding protein (ICSBP). In addition, ICSBP also regulated COL4A1 and COL4A2.

Confirmatory analysis. A total of 2,164 DEGs were obtained from skeletal muscle after $1 \mathrm{~h}$ of one-legged exercise, including 809 upregulated genes and 1,355 downregulated genes. There were 53 overlapping DEGs in the two datasets, such as VEGFA and FGF6 (Fig. 7; Table SI). In the 'GSE41769' dataset, collagen type VIII $\alpha 2$ Chain and collagen type II $\alpha 1$ chain (COL2A1) were differentially expressed after $1 \mathrm{~h}$ of one-legged exercise. However, in the 'GSE109657' dataset, COL4A1 and COL4A2 were differentially expressed after HIIT, suggesting that exercise may induce expression changes of collagen-associated genes.

In addition, the DEGs were significantly enriched in 88 KEGG pathways and numerous GO_BPs, including the PI3K-Akt signaling pathway (involving COL2A1 and VEGFA), regulation of angiogenesis, sprouting angiogenesis, regulation of extracellular matrix assembly, extracellular matrix organization and focal adhesion assembly (Table SII). Furthermore, these results were consistent with the results from the analysis of genes in the brown module (Table II).

\section{Discussion}

In the present study, a total of 530 genes were found to be abnormally expressed in skeletal muscle after a 6-week HIIT, suggesting an effect of HIIT on the skeletal muscle. In total, three significant modules (brown, blue and red modules) were identified after WGCNA, and the genes, COL4A1 and COL4A2, in module brown showed the strongest correlation with HIIT. There were 106, 74 and 49 DEGs in the brown, blue and red modules, respectively, which were significantly enriched in focal adhesion, extracellular matrix organization 
Table III. Nodes in the protein-protein interaction network with degree $>5$.

\begin{tabular}{|c|c|c|c|}
\hline Nodes & Regulation & Module & Degree \\
\hline EGFR & down & blue & 23 \\
\hline VEGFA & up & blue & 19 \\
\hline AR & down & red & 14 \\
\hline KIT & up & brown & 12 \\
\hline KDR & up & brown & 11 \\
\hline PECAM1 & up & brown & 11 \\
\hline PKM & up & brown & 11 \\
\hline CYP3A4 & down & red & 10 \\
\hline MDH1 & up & blue & 9 \\
\hline CD38 & down & blue & 9 \\
\hline COL4A1 & up & brown & 8 \\
\hline CDH5 & up & brown & 8 \\
\hline COL4A2 & up & brown & 7 \\
\hline LAMB1 & up & brown & 7 \\
\hline FBP2 & up & brown & 7 \\
\hline CARM1 & down & brown & 7 \\
\hline NRP1 & up & brown & 6 \\
\hline GOT1 & up & blue & 6 \\
\hline ADH1B & down & blue & 6 \\
\hline LAMA4 & up & brown & 6 \\
\hline CYP1A1 & up & red & 6 \\
\hline PFKM & up & brown & 6 \\
\hline APLNR & up & blue & 6 \\
\hline FGF6 & up & red & 6 \\
\hline PGAM2 & up & brown & 6 \\
\hline MCAM & up & red & 6 \\
\hline MGST1 & down & blue & 6 \\
\hline GSTM5 & down & brown & 6 \\
\hline CKMT2 & up & blue & 6 \\
\hline FCGR1A & down & brown & 6 \\
\hline ADH1C & down & blue & 5 \\
\hline GNG11 & up & brown & 5 \\
\hline IL6R & down & red & 5 \\
\hline HSPB1 & down & brown & 5 \\
\hline MYH1 & down & blue & 5 \\
\hline CD93 & up & brown & 5 \\
\hline
\end{tabular}

and the PI3K-Akt signaling pathway. Furthermore, it was found that VEGFA, COL4A1 and COL4A2 were the hub genes in the PPI network, and were all regulated by $\mathrm{miR}-29 \mathrm{a} / \mathrm{b} / \mathrm{c}$. Therefore, the present results indicated that these genes, together with miR-29a/b/c, may have a regulatory function in the skeletal muscle response to HIIT.

VEGFA is a protein-coding gene that plays a crucial role in vascular endothelial cell growth and angiogenesis (21). Gustafsson et al (22), indicated that exercise can promote the expression of VEGFA involved in the non-pathological angiogenesis in human skeletal muscle. Moreover, Baum et al (23) showed that exercise training induces ultrastructural changes, such as pericyte mobilization and basement membrane

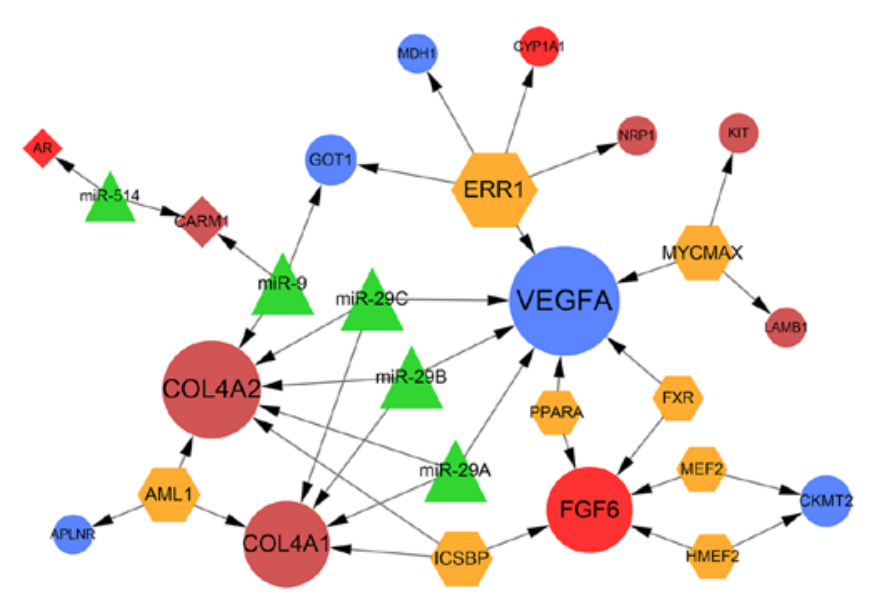

Figure 6. miRNA-TF-target regulatory network. A circle represents upregulated DEGs; a rhombus represents downregulated DEGs; green triangles represent miRNAs; yellow hexagons represent TFs. Brown, blue and red represent the DEGs belonging to the brown, blue and red modules, respectively. miRNA, microRNA; TFs, transcription factors; DEGs, differentially expressed genes.

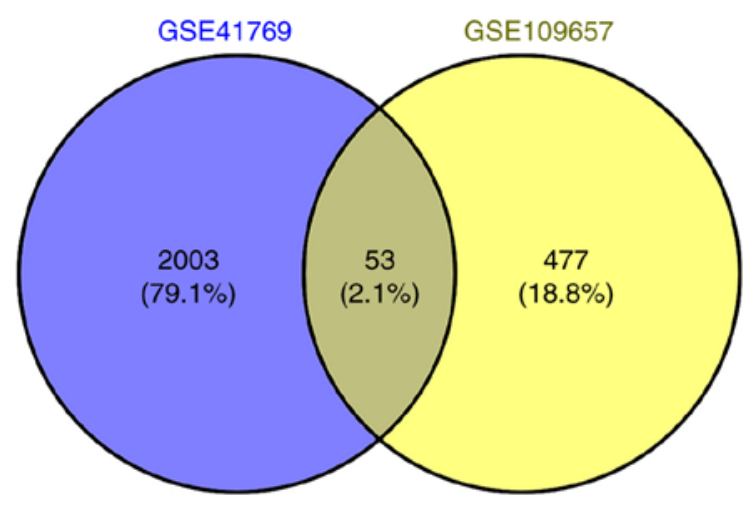

Figure 7. A total of 53 overlapping differentially expressed genes in GSE41769 and GSE109657 datasets. Venn diagram to identify the overlapped DEGs in the two datasets.

thinning in the capillaries, and this process is associated with exercise-induced angiogenesis. The generation of new capillaries in skeletal muscles is an adaptive response of the skeletal muscle to exercise (24). Capillaries serve as major sites for the transport of gas, nutrients and metabolic waste; exercise-induced capillary angiogenesis ensures that the increased need of active skeletal muscle for oxygen and nutrients is met (24). The present results suggested that VEGFA was upregulated in skeletal muscles after HIIT, which was consistent with results from previous studies $(23,24)$, suggesting that skeletal muscle angiogenesis was induced after HIIT and is associated with the upregulation of VEGFA.

Previous studies in animal models have shown that local VEGFA gene transfer accelerates long-term angiogenesis $(25,26)$. However, unregulated VEGFA expression results in adverse changes leading to aberrant muscle morphology (27), suggesting the need for the regulation of VEGFA expression in long-term gene transfer cases. Klagsbrun (28) revealed that the extracellular matrix is a critical component in the regulation of angiogenesis and could also provide a barrier to angiogenesis. Furthermore, Sottile (29) reported that the extracellular matrix 
controls the growth, differentiation and migration of vascular endothelial cells in the course of angiogenesis. Moreover, remodeling of extracellular matrix results in events that either promote or inhibit angiogenesis (29). Focal adhesion also participates in regulating cell migration and proliferation during angiogenesis, and adhesion molecules may interact with the extracellular matrix to exert an effect $(30,31)$. The $\mathrm{PI} 3 \mathrm{~K} / \mathrm{Akt}$ signaling pathway is reported to regulate vascular endothelial cell elongation and endothelial capillary stability during angiogenesis $(32,33)$. In the present study, COL4A1 and COL4A2 were significantly enriched in focal adhesion, extracellular matrix organization and the PI3K/Akt signaling pathway. COL4A1 and COL4A2 are type IV collagen $\alpha$ proteins, and are major components of the basement membrane (34). COL4A1 mutations are reported to cause the endothelial cell defects and apoptosis in the capillaries of skeletal muscle (35). Therefore, COL4A1 and COL4A2 may mediate the growth and migration of vascular endothelial cells via cell adhesion, extracellular matrix organization and the PI3K/Akt signaling pathway, and as a result can regulate exercise-induced skeletal muscle angiogenesis.

In the present study, miR-29a/b/c were predicted to regulate VEGFA, COL4A1 and COL4A2 in the regulatory network. A previous study showed that miR-29 plays an important role in regulating skeletal muscle growth and differentiation via decreasing Akt3 (36). Furthermore, it was demonstrated that miR-29b mediates the expression of collagen type I $\alpha$ via the PI3K/Akt signaling pathway in human Tenon's fibroblasts (37). In addition, miR-29b targets VEGFA via the PI3K/Akt signaling pathway to suppress angiogenesis in endometrial carcinoma (38). Moreover, miR-29c and miR-29a are crucial regulators in the cell cycle progression and growth, as well as in the angiogenic properties of human umbilical vein endothelial cells $(39,40)$. The present study identified the potential roles of $\mathrm{miR}-29 \mathrm{a} / \mathrm{b} / \mathrm{c}$ in skeletal muscle and angiogenesis. Therefore, miR-29a/b/c may regulate the exercise-induced angiogenesis in skeletal muscle by targeting VEGFA, COL4A1 and COL4A2 via the PI3K/Akt signaling pathway. However, further experimental studies are required to investigate the present results in greater depth.

In conclusion, the present results suggested that VEGFA, COL4A1 and COL4A2 were upregulated in the skeletal muscle in response to HIIT. Furthermore, COL4A1 and COL4A2 may mediate the growth and migration of vascular endothelial cells via cell adhesion and extracellular matrix organization, along with the regulation of angiogenesis. It was demonstrated that skeletal muscle angiogenesis may be regulated by $\mathrm{miR}-29 \mathrm{a} / \mathrm{b} / \mathrm{c}$ targeting VEGFA, COL4A1 and COL4A2 via the PI3K/Akt signaling pathway. Therefore, the present results may facilitate continued investigation into the effect of exercise on skeletal muscles.

\section{Acknowledgements}

Not applicable.

\section{Funding}

No funding was received.

\section{Availability of data and materials}

All data generated or analyzed during this study are included in this published article.

\section{Authors' contributions}

LC was responsible for the conception and design of the research, and drafting the manuscript. JB performed the data acquisition. YL performed the data analysis and interpretation. LC and JB participated in the design of the study and performed the statistical analysis. All authors have read and approved the final manuscript.

\section{Ethics approval and consent to participate}

Not applicable.

\section{Patient consent for publication}

Not applicable.

\section{Competing interests}

The authors declare that they have no competing interests.

\section{References}

1. Horak M, Novak J and Bienertova-Vasku J: Muscle-specific microRNAs in skeletal muscle development. Dev Biol 410: 1-13, 2016.

2. Egan B and Zierath JR: Exercise metabolism and the molecular regulation of skeletal muscle adaptation. Cell Metab 17: 162-184, 2013.

3. Drake JC, Wilson RJ and Yan Z: Molecular mechanisms for mitochondrial adaptation to exercise training in skeletal muscle. FASEB J 30: 13-22, 2016

4. Tunstall RJ, Mehan KA, Wadley GD, Collier GR, Bonen A, Hargreaves M and Cameron-Smith D: Exercise training increases lipid metabolism gene expression in human skeletal muscle. Am J Physiol Endocrinol Metab 283: E66-E72, 2002.

5. Nieman DC, Shanely RA, Zwetsloot KA, Meaney MP and Farris GE: Ultrasonic assessment of exercise-induced change in skeletal muscle glycogen content. BMC Sports Sci Med Rehabil 7: 9, 2015.

6. Hoppeler H: Exercise-induced ultrastructural changes in skeletal muscle. Int J Sports Med 7: 187-204, 1986.

7. Baar K, Wende AR, Jones TE, Marison M, Nolte LA, Chen M, Kelly DP and Holloszy JO: Adaptations of skeletal muscle to exercise: Rapid increase in the transcriptional coactivator PGC-1. FASEB J 16: 1879-1886, 2002.

8. Cantó C, Jiang LQ, Deshmukh AS, Mataki C, Coste A, Lagouge M,Zierath JR and Auwerx J: Interdependence of AMPK and SIRT1 for metabolic adaptation to fasting and exercise in skeletal muscle. Cell Metab 11: 213-219, 2010.

9. Tzanis G, Philippou A, Karatzanos E, Dimopoulos S, Kaldara E, Nana E, Pitsolis T, Rontogianni D, Koutsilieris $\mathrm{M}$ and Nanas S: Effects of high-intensity interval exercise training on skeletal myopathy of chronic heart failure. J Card Fail 23: 36-46, 2017.

10. Peake JM, Della Gatta P, Suzuki K and Nieman DC: Cytokine expression and secretion by skeletal muscle cells: Regulatory mechanisms and exercise effects. Exerc Immunol Rev 21: 8-25, 2015.

11. McCarthy JJ: microRNA and skeletal muscle function: Novel potential roles in exercise, diseases, and aging. Front Physiol 5: 290-290, 2014.

12. Xu Y, Zhao C, Sun X, Liu Z and Zhang J: MicroRNA-761 regulates mitochondrial biogenesis in mouse skeletal muscle in response to exercise. Biochem Biophys Res Commun 467: 103-108, 2015. 
13. Sasaki T, Kuboyama A, Mita M, Murata S, Shimizu M, Inoue J, Mori $\mathrm{K}$ and Sato R: The exercise-inducible bile acid receptor Tgr5 improves skeletal muscle function in mice. J Biol Chem 293: 10322-10332, 2018.

14. Henríquez-Olguín C, Renani LB, Arab-Ceschia L, Raun SH, Bhatia A, Li Z, Knudsen JR, Holmdahl R and Jensen TE: Adaptations to high-intensity interval training in skeletal muscle require NADPH oxidase 2. Redox Biol 24: 101188, 2019.

15. Miyamoto-Mikami E, Tsuji K, Horii N, Hasegawa N, Fujie S, Homma T, Uchida M, Hamaoka T, Kanehisa H, Tabata I, et al: Gene expression profile of muscle adaptation to high-intensity intermittent exercise training in young men. Sci Rep 8: $16811-16811,2018$

16. Song WM and Zhang B: Multiscale embedded gene co-expression network analysis. PLOS Comput Biol 11: e1004574, 2015.

17. Ritchie ME, Phipson B, Wu D, Hu Y, Law CW, Shi W and Smyth GK: limma powers differential expression analyses for RNA-sequencing and microarray studies. Nucleic Acids Res 43 : e47, 2015 .

18. Langfelder $\mathrm{P}$ and Horvath S: WGCNA: An R package for weighted correlation network analysis. BMC Bioinformatics 9: $559,2008$.

19. Ni M, Liu X, Wu J, Zhang D, Tian J, Wang T, Liu S, Meng Z, Wang K, Duan X, et al: Identification of candidate biomarkers correlated with the pathogenesis and prognosis of non-small cell lung cancer via integrated bioinformatics analysis. Front Genet 9: 469, 2018.

20. Catoire M, Mensink M, Boekschoten MV, Hangelbroek R, Müller M, Schrauwen P and Kersten S: Pronounced effects of acute endurance exercise on gene expression in resting and exercising human skeletal muscle. PLoS One 7: e51066, 2012.

21. Teng ACT, Kuraitis D, Deeke SA, Ahmadi A, Dugan SG, Cheng BLM, Crowson MG, Burgon PG, Suuronen EJ, Chen HH,etal: IRF2BP2 is a skeletal and cardiac muscle-enriched ischemia-inducible activator of VEGFA expression. FASEB J 24: 4825-4834, 2010.

22. Gustafsson T, Rundqvist H, Norrbom J, Rullman E, Jansson E and Sundberg CJ: The influence of physical training on the angiopoietin and VEGF-A systems in human skeletal muscle. J Appl Physiol 1985 103: 1012-1020, 2007.

23. Baum O, Gübeli J, Frese S, Torchetti E, Malik C, Odriozola A Graber F, Hoppeler H and Tschanz SA: Angiogenesis-related ultrastructural changes to capillaries in human skeletal muscle in response to endurance exercise. J Appl Physiol 1985 119: $1118-1126,2015$

24. Haas TL and Nwadozi E: Regulation of skeletal muscle capillary growth in exercise and disease. Appl Physiol Nutr Metab 40: 1221-1232, 2015.

25. Morland C, Andersson KA, Haugen ØP, Hadzic A, Kleppa L, Gille A, Rinholm JE, Palibrk V, Diget EH, Kennedy LH, et al: Exercise induces cerebral VEGF and angiogenesis via the lactate receptor HCAR1. Nat Commun 8: 15557, 2017.

26. Palazon A, Tyrakis PA, Macias D, Velica P, Rundqvist H, Fitzpatrick S, Vojnovic N, Phan AT, Loman N, Hedenfalk I, et al: An HIF-1 $\alpha$ /VEGF-A axis in cytotoxic T cells regulates tumor progression. Cancer Cell 32: 669-683.e5, 2017.
27. Karvinen H, Pasanen E, Rissanen TT, Korpisalo P, Vähäkangas E, Jazwa A, Giacca M and Ylä-Herttuala S: Long-term VEGF-A expression promotes aberrant angiogenesis and fibrosis in skeletal muscle. Gene Ther 18: 1166-1172, 2011.

28. Klagsbrun M: Regulators of angiogenesis: Stimulators, inhibitors, and extracellular matrix. J Cell Biochem 47: 199-200, 1991.

29. Sottile J: Regulation of angiogenesis by extracellular matrix BBA-Reviews on Cancer 1654: 13-22, 2004.

30. Zhao X and Guan JL: Focal adhesion kinase and its signaling pathways in cell migration and angiogenesis. Adv Drug Deliv Rev 63: 610-615, 2011.

31. Wary KK, Kohler EE and Chatterjee I: Focal adhesion kinase regulation of neovascularization. Microvasc Res 83: 64-70, 2012.

32. Tsuji-Tamura K and Ogawa M: Inhibition of the PI3K-Akt and mTORC1 signaling pathways promotes the elongation of vascular endothelial cells. J Cell Sci 129: 1165-1178, 2016.

33. Lee NY, Golzio C, Gatza CE, Sharma A, Katsanis N and Blobe GC: Endoglin regulates PI3-kinase/Akt trafficking and signaling to alter endothelial capillary stability during angiogenesis. Mol Biol Cell 23: 2412-2423, 2012.

34. Mao M, Alavi MV, Labelle-Dumais C, Gould DB and Type IV: Type IV collagens and basement membrane diseases: Cell biology and pathogenic mechanisms. Curr Top Membr 76: 61-116, 2015.

35. Guiraud S, Migeon T, Ferry A, Chen Z, Ouchelouche S, Verpont MC, Sado Y, Allamand V, Ronco P and Plaisier E: HANAC Col4a1 mutation in mice leads to skeletal muscle alterations due to a primary vascular defect. Am J Pathol 187: 505-516, 2017.

36. Wei W, He HB, Zhang WY, Zhang HX, Bai JB, Liu HZ, Cao JH, Chang KC, Li XY and Zhao SH: miR-29 targets Akt3 to reduce proliferation and facilitate differentiation of myoblasts in skeletal muscle development. Cell Death Dis 4: e668, 2013.

37. Li N, Cui J, Duan X, Chen H and Fan F: Suppression of type I collagen expression by miR-29b via PI3K, Akt, and Sp1 pathway in human Tenon's fibroblasts. Invest Ophthalmol Vis Sci 53: 1670-1678, 2012.

38. Chen HX, Xu XX, Tan BZ, Zhang Z and Zhou XD: MicroRNA-29b inhibits angiogenesis by targeting VEGFA through the MAPK/ERK and PI3K/Akt signaling pathways in endometrial carcinoma. Cell Physiol Biochem 41: 933-946, 2017.

39. Hu Y, Deng F, Song J, Lin J, Li X, Tang Y, Zhou J, Tang T and Zheng L: Evaluation of miR-29c inhibits endotheliocyte migration and angiogenesis of human endothelial cells by suppressing the insulin like growth factor 1 . Am J Transl Res 7: 489-501, 2015.

40. Yang Z, Wu L, Zhu X, Xu J, Jin R, Li G and Wu F: MiR-29a modulates the angiogenic properties of human endothelial cells. Biochem Biophys Res Commun 434: 143-149, 2013.

This work is licensed under a Creative Commons Attribution-NonCommercial-NoDerivatives 4.0 International (CC BY-NC-ND 4.0) License. 